\title{
SYNTHESIS, IN SILICO STUDIES AND EVALUATION OF ANTIBACTERIAL ACTIVITY OF 4-SUBSTITUTED BENZYLIDENE-2-(PHENOXYMETHYL) OXAZOL-5(4H)-ONES
}

\author{
SHAHEEN BEGUM ${ }^{1 *}$, ARIFA BEGUM SK ${ }^{2}$, MANEESHA M $^{1}$, OME SHANTHI ${ }^{1}{ }^{1}$, SATYA VARALAKSHMI D ${ }^{1}$, \\ MANJUSHA RK ${ }^{1}$, BHARATHI $\mathrm{K}^{1}$
}

${ }^{1}$ Institute of Pharmaceutical Technology, Sri Padmavati Mahila Visvavidyalayam (Women's University), Tirupati, Andhra Pradesh, India. ${ }^{2}$ Avanti College of Pharmaceutical Sciences, Guntapally, Hyderabad, Telangana, India. Email: shaheen.pharmchem@gmail.com

Received: 15 December 2018, Revised and Accepted: 10 April 2019

ABSTRACT

Objective: Arylidene-1, 3-oxazol-5-ones represent potential antibacterial agents. In the present work, a series of 4-substituted benzylidene-2(phenoxymethyl) oxazol-5(4H)-ones were synthesized and screened for antibacterial activity against Gram-negative bacteria Escherichia coli. To explore plausible mechanisms, synthesized compounds were docked with DNA-Gyrase enzyme.

Methods: All the reactants, phenoxy acetyl chloride, acetic anhydride, sodium acetate, substituted aromatic aldehydes, and glycine were triturated in a mortar by mechanical stirring. The antibacterial potentiality of the compounds was screened against $E$. coli using the disk diffusion method and the activity was recorded as a zone of inhibition.

Results: Compound 2d, possessing 3, 4, 5-trimethoxy functionality on benzylidene ring exhibited the highest activity with 19 mm of the zone of inhibition which might be due to its higher interactions with DNA-Gyrase enzyme ( $\Delta \mathrm{G}-8.41 \mathrm{kcal} / \mathrm{mol})$. Compounds $2 \mathrm{a}, 2 \mathrm{~b}$, and $2 \mathrm{c}$ exhibited moderate activity in the antimicrobial assay as well as in docking study indicating the positive contribution of substitution on benzylidene ring.

Conclusion: A series of 4-substituted benzylidene-2-(phenoxymethyl) oxazol-5(4H)-ones were synthesized and evaluated for antibacterial activity. Compounds $2 \mathrm{a}, 2 \mathrm{~b}$, and $2 \mathrm{c}$ displayed moderate activity whereas $2 \mathrm{~d}$ showed maximum zone of inhibition ( $19 \mathrm{~mm})$. The good activity of these derivatives presumed to be due to the conformational flexibility of phenoxy methylene moiety which can be well accommodated in the target binding site.

Keywords: 4-Benzylidene-2-(phenoxymethyl) oxazol-5(4H)-ones, Molecular docking, DNA-Gyrase, Cup-plate method, Molecular properties.

(C) 2019 The Authors. Published by Innovare Academic Sciences Pvt Ltd. This is an open access article under the CC BY license (http://creativecommons. org/licenses/by/4. 0/) DOI: http://dx.doi.org/10.22159/ajpcr.2019.v12i5.31390

\section{INTRODUCTION}

According to the recent reports, 30 new infectious diseases and existing infections including HIV, malaria, and tuberculosis are re-emerging globally at an alarming rate. Bacterial infections permeate developing countries, and in particular infants, children are seriously affected. Eravacycline and omadacycline are tetracycline antibiotics highly effective in bacterial skin and pneumonia infections $[1,2]$. The greatest hurdle with the existing antibacterial agents is bacterial resistance due to inappropriate usage of antibiotics, lack of immunity in human beings and fast genetic mutations in bacteria. In spite of all the available antibacterial agents, millions of deaths have been recorded due to malarial infections, tuberculosis, diarrhea, and respiratory infections. These data motivate researchers to develop efficacious antibacterial agents [3-6].

Heterocyclics such as quinoline, indoles, oxadiazoles, triazoles, thiazines, pyridazines, pyrazoles, and oxazoles display noticeable antibacterial activity against different pathological bacteria [7-11] Oxazol-5-ones have been extensively studied for their expansive biological activities, including anti-inflammatory, antidiabetic, anticancer, antimicrobial, antitumor, antiviral, and anti-HIV activities [11-15]. Interestingly, 1, 3-oxazol-5-one containing compound competitively inhibited Rhoassociated coiled-coil-containing protein kinase 2, a kinase which is pivotal in calcium ion sensitization in vascular smooth muscle [16]. Diverse set of pharmacological activities of this heterocyclic moiety might be due to to its ability to act as hydrogen bond acceptor (HBA) and to form non-bonded interactions with the target site amino acids. Noticeably, oxazolones linked to arylidene moiety display potent antimicrobial activity [17-19]. The presence of arylidene linkage modulates/enhances the biological activity of oxazolones by altering the lipophilic and steric properties [20,21]. Substituted 4-benzylidene-2phenyloxazol-5(4H)-ones (Fig. 1) display potent antimicrobial activity against various Gram-negative and Gram-positive microorganisms, [18] and the structure-activity relationship of the studies revealed that the presence of methoxy group on benzylidene ring enhances the antibacterial activity.

Phenoxy acid moiety is found as a core scaffold in several antibacterial compounds $[22,23]$. Substituted phenoxymethyl oxazolones linked to arylidene moiety (Fig. 2) were synthesized by Aaglawe et al. In their study, it was demonstrated that substituent groups on benzylidene ring (4-chloro/4-bromo) exert a significant effect on antimicrobial activity (against Escherichia coli) [24]. In view of the potent antimicrobial activity of these compounds and to explore the influence of other functional groups (4-fluorine, 4-hydroxy, 3, 4, 5-trimethoxy, 4-dimethyamino, 4-diethylamino) on the antimicrobial activity of phenoxymethyl oxazolones, it was planned to synthesize few 4-substituted benzylidene-2-(phenoxymethyl) oxazol-5(4H)-ones. The synthesized compounds were evaluated for their antimicrobial potentiality using E. coli.

Mechano heterocyclic chemistry involves applying mechanical energy such as grinding (grindstone technique) which accelerates the rate of reaction. The multicomponent approach provides simple procedures to synthesize complex structures [25-27].

\section{METHODS}

"Thermonik Precision Melting point cum boiling point apparatus, model C-PMB-2 was used for melting point determination and values are uncorrected. Phenoxy acetic acid and acetic anhydride 


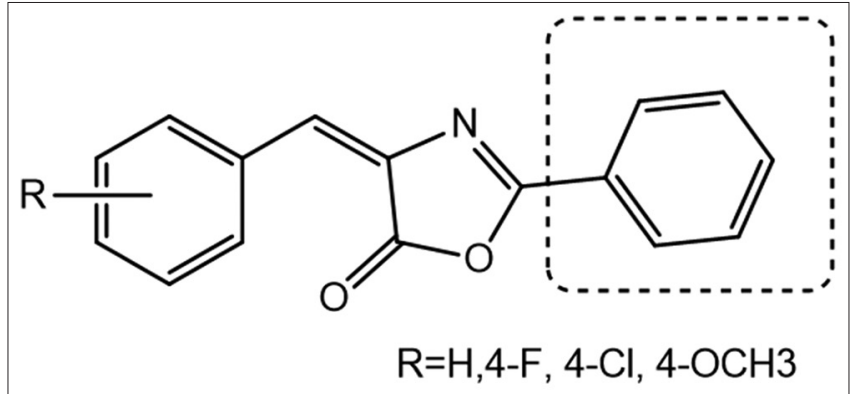

Fig. 1: 4-Substituted benzylidene-2-phenyloxazol-5(4H)-ones

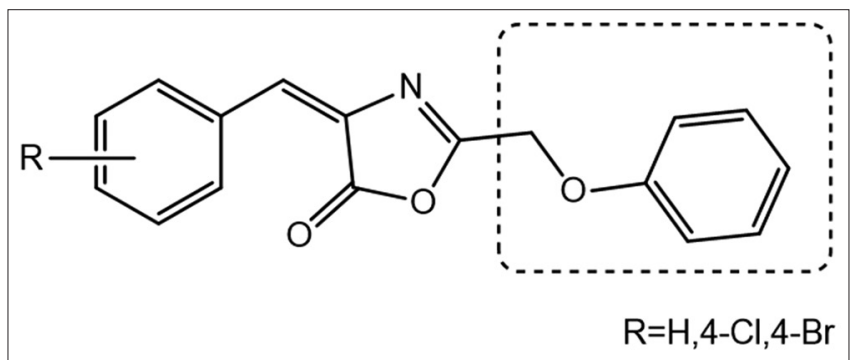

Fig. 2: 4-Substituted benzylidene-2-(phenoxymethyl) oxazol-5(4H)-ones

were purchased from Sigma-Aldrich chemicals, and other chemicals were from Merck and HiMedia. Microorganisms were obtained from the Faculty of Microbiology, Sri Padmavati Mahila Visvavidyalayam, Tirupati, India. Thin-layer chromatography (TLC) was used to observe the reaction and to check the purity of synthesized compounds. Iodine chamber was used to develop spots. Infrared (IR) spectral studies were performed using $\mathrm{KBr}$ disks on Perkin Elmer Spectrum BX-I IR spectrophotometer $\left(\mathrm{cm}^{-1}\right)$. The proton nuclear magnetic resonance $\left({ }^{1} \mathrm{H}\right.$ NMR) spectra were obtained by dissolving the samples in $\mathrm{CDCl}_{3}$ on JOEL-400 instrument. Mass (m/z) spectra were obtained using LCMS-8030 Mass Spectrometer (mass selective detector). The title compounds (2a-2f) were recrystallized twice from alcohol and water mixture.

General procedure for the synthesis of 4-substituted benzylidene2-(phenoxymethyl) oxazol-5(4H)-one

Synthesis of 4-benzylidene-2-(phenoxymethyl) oxazol-5(4H)-one

Step 1: Synthesis of phenoxy acid chloride (1)

Phenoxy acetic acid $(0.01 \mathrm{~mol})$ and thionyl chloride $(0.01 \mathrm{~mol})$ were mixed properly and refluxed for $2 \mathrm{~h}$. Excess of thionyl chloride was removed by applying vacuum distillation [28].

Step 2: Synthesis of 4-benzylidene-2-(phenoxymethyl) oxazol-5(4H)one

Equimolar amounts of glycine, 4-fluorobenzaldehyde, phenoxy acetyl chloride, fused sodium acetate, and few drops of acetic anhydride were triturated in a porcelain mortar and pestle for 15-30 min [27]. Completion of the reaction was monitored by TLC; the resultant solid was washed with cold water and recrystallized from ethanol and water mixture to obtain crude compound (2a).

Similarly, other 4-substituted benzylidene-2-(phenoxymethyl) oxazol$5(4 \mathrm{H})$-ones $(2 \mathrm{~b}-2 \mathrm{f})$ were synthesized following a similar procedure where substituted benzaldehydes were used in place of 4-fluoro benzaldehyde (Fig. 3)

\section{Antimicrobial activity}

The antibacterial activity was tested against E. coli by the cup-plate method.

\section{Preparation of agar media}

Agar medium was prepared by mixing meat extract $(0.9 \mathrm{~g})$, peptone $(1.5 \mathrm{~g})$, and agar ( $5 \mathrm{~g})$ in $100 \mathrm{ml}$ of distilled water. The antibiotic amikacin was used to compare the activities of the test compounds against various bacteria. Amikacin activity was screened by preparing specific media which includes agar $(2 \mathrm{~g})$, peptone $(0.6 \mathrm{~g})$, yeast extract $(0.3 \mathrm{~g})$, and beef extract $(0.15 \mathrm{~g})$ in $100 \mathrm{ml}$ distilled water.

\section{Preparation of test compounds}

Stock solutions (2a-f) were prepared by dissolving $5 \mathrm{mg} / \mathrm{mL}$ of the title compounds in dimethyl sulfoxide (DMSO), and from these, $50 \mu \mathrm{L}$ and $100 \mu \mathrm{L}$ test solutions were prepared. DMSO was used as negative control. Tests, as well as negative control, were added with the help of sterile pipettes.

\section{Procedure}

A small quantity of microbial suspension was added to the agar media $\left(45-50^{\circ} \mathrm{C}\right)$, and agar media were immediately transferred to sterile Petri plates. When media were settled, around $4 \mathrm{~mm}$ plugs of the media were removed using a sterile corn borer into each Petri plate. Test solutions of exact volume were added to the holes and incubated for $24 \mathrm{~h}$. The zone of inhibition was measured in millimeters after $24 \mathrm{~h}$. Each test was conducted as triplicates and the average zone of inhibition was considered.

Prediction of molecular and absorption, distribution, metabolism, and excretion (ADME) descriptors

Prediction of molecular, pharmacokinetic properties, and toxicity is inevitable in the current drug development process. Log $\mathrm{P}$ (lipophilicity), HBD (number of hydrogen bond donors), HBA, topological polar surface area as well as the bioactivity scores, i.e., affinity of the compounds for various targets such as G-protein coupled receptor, ion channel, kinase, nuclear receptor, protease, and enzyme were predicted using online tool (http://www. molinspiration. com/ cgi-bin/properties). ADME properties of the title compounds were predicted using the preADMET online server (http://preadmet.bmdrc. org/). Various ADME properties including $\mathrm{CaCo} 2$ cell permeability, human intestinal absorption, plasma protein binding, blood-brain barrier penetration, skin permeability, and Maden Darby canine kidney cell permeability were predicted.

\section{Swiss dock studies}

Molecular docking studies were performed by Swiss Dock, a userfriendly tool. The protein and ligand structures can be directly submitted for docking and results of the docking studies can be viewed by chimera $[29,30]$.

Protein

The crystal structure of DNA-Gyrase subunit B was retrieved from the protein data bank (PDB entry: $1 \mathrm{KZN}$ ). Chain A was selected for the docking study which includes 205 amino acids. Ligand structures were submitted in mol.2 form and the results were viewed in chimera.

\section{Ligands}

Ligand structures (Figs. 4 and 5) were submitted in mol.2 forms.

\section{RESULTS AND DISCUSSION}

Chemistry

A series of 4-substituted benzylidene-2-(phenoxymethyl) oxazol$5(4 \mathrm{H})$-ones were synthesized by triturating phenoxy acetyl chloride, sodium acetate, acetic anhydride, glycine, and substituted benzaldehyde in a mortar (mechanochemical grinding method). Compounds were obtained in good yields (60-78\%). Title compounds were obtained within 15-30 min. The synthesized compounds were purified by recrystallization using an aqueous-alcoholic mixture. The physical data of the compounds are given in Table 1 . The structures of the final compounds were characterized by spectral data including UV, IR, ${ }^{1} \mathrm{H}$ NMR, and mass spectral analysis. 


\section{Step1. Synthesis of phenoxy acid chloride}

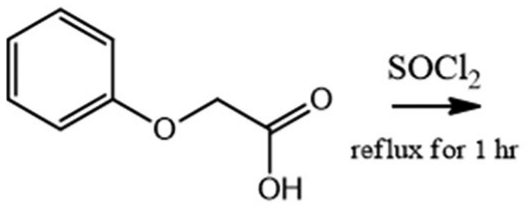<smiles>O=C(Cl)COc1ccccc1</smiles>

Phenoxy acetic acid

Phenoxy acetylchloride

(1)

\section{Step.2 Synthesis of 4-substituted benzylidene-2-(phenoxymethyl) oxazol-5(4H)-one}<smiles>CC(=O)O[C+](C)C(=O)[NH2+][18O]C(C)=O</smiles>

Acetic anhydride sodium acetate glycine<smiles>O=C(Cl)COc1ccccc1</smiles><smiles>[R]c1cccc(C=O)c1</smiles><smiles>[R]c1cccc(/C=C2/N=C(COc3ccccc3)OC2=O)c1</smiles>

Phenoxy acetyl chloride

Substituted benzaldehyde

$\mathrm{R}=\mathrm{F}, \mathrm{NO}_{2}, \mathrm{OH}, 3,4,5-\left(\mathrm{OCH}_{3}\right)_{3}, 4-\mathrm{N}\left(\mathrm{CH}_{3}\right), 4-\mathrm{N}\left(\mathrm{C}_{2} \mathrm{H}_{5}\right)_{2}$

Fig. 3: Scheme for the synthesis of 4-substituted benzylidene-2-(phenoxymethyl) oxazol-5(4H)-ones (2a-2f)

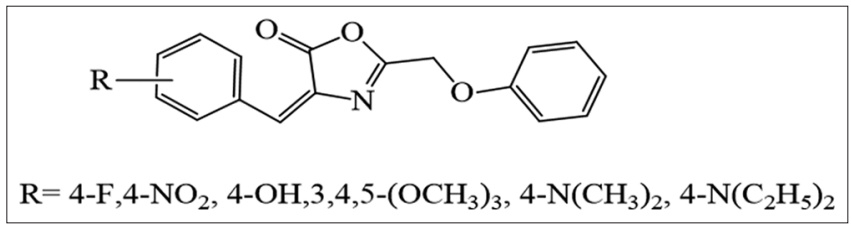

Fig. 4: 4-Substituted benzylidene-2-(phenoxymethyl) oxazol$5(4 \mathrm{H})$-ones $(2 \mathrm{a}-2 \mathrm{f})$

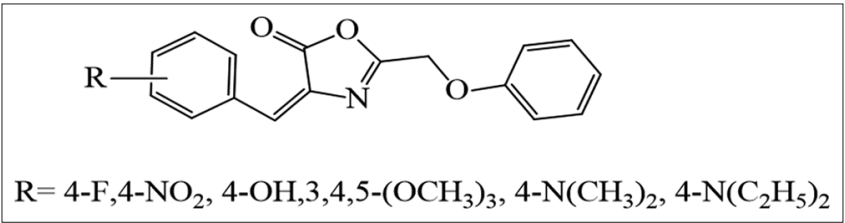

Fig. 5: 4-Substituted benzylidene-2-phenyloxazol-5(4H)-ones (1-6)

IR spectra of the compounds showed bands at 2950-2900 due to $\mathrm{C}-\mathrm{H}$ aromatic; $2850-2800$ due to $\mathrm{C}-\mathrm{H}$ aliphatic; $1760-1720 \mathrm{~cm}^{-1}$ due to $\mathrm{C}=0$ in the lactone ring; and $1650-1600 \mathrm{~cm}^{-1}$ due to $\mathrm{C}=\mathrm{C} .{ }^{1} \mathrm{H}$ NMR data clearly indicated the presence of phenoxy methylene protons at around 4.0-4.5 ppm and aromatic protons were observed around 6.42-7.89 ppm. Mass spectrum of the compounds showed characteristic peaks $\left(\mathrm{M}^{+}\right)$
General structure of the synthesized compounds<smiles>[R]c1cccc(/C=C2/N=C(COc3ccccc3)OC2=O)c1</smiles>

$\mathrm{R}=4-\mathrm{F}, 4-\mathrm{NO}_{2}, 4-\mathrm{OH}, 3,4,5-\left(\mathrm{OCH}_{3}\right)_{3}, 4-\mathrm{N}\left(\mathrm{CH}_{3}\right)_{2}, 4-\mathrm{N}\left(\mathrm{C}_{2} \mathrm{H}_{5}\right)_{2}$

Physicochemical data of compounds 2a-2f

2a: 4-(4-fluorobenzylidene)-2-(phenoxymethyl) oxazol-5(4H)-one Fourier-transform IR (FTIR) (KBr) $\mathrm{cm}^{-1:} 2958.78$ (C-H aromatic str), 2855.12 (C-H aliphatic stretch), 1735.78 ( $\mathrm{C}=0$ str of lactone ring), 1665.20 ( $\mathrm{C}=\mathrm{C}$ str), 870.25 (C-H aromatic bend). ${ }^{1} \mathrm{H}$ NMR $\left(\mathrm{CDCl}_{3}\right): \delta 4.08\left(\mathrm{~s}, 2 \mathrm{H},-\mathrm{OCH}_{2}-\right.$ ), 7.01-7.85 (m, 10H, Ar-H\&=CH-) NMR $\left(\mathrm{CDCl}_{3}\right)$ MS (m/z): $298.28[\mathrm{M}+1]^{+}$ Elemental Analysis: C, 68.76; H, 4.06; F, 6.38; N, 4.74; 0, 16.14 .

2b: 4-(4-nitrobenzylidene)-2-(phenoxymethyl) oxazol-5(4H)-one FTIR (KBr) cm ${ }^{-1}: 2958.35$ (C-H aromatic str), 2918.69 (C-H aliphatic str), 1760.11 (C=0 str of lactone ring), 1671.40 (C=C str), 831.43 (C-H aromatic bend). ${ }^{1} \mathrm{H}$ NMR $\left(\mathrm{CDCl}_{3}\right): \delta 4.12\left(\mathrm{~s}, 2 \mathrm{H},-\mathrm{OCH}_{2}-\right), 6.75-7.48$ (m, 10H, Ar-H\&=CH-) MS (m/z): $338.3[\mathrm{M}]^{+}$.

2c: 4-(4-hydroxybenzylidene)-2-(phenoxymethyl) oxazol-5(4H)-one FTIR (KBr) cm ${ }^{-1}: 2998.14$ (C-H aromatic str), 2875.19 (C-H aliphatic str), $1724.35(\mathrm{C}=0$ str of lactone ring), 1665.32 ( $\mathrm{C}=\mathrm{C}$ str), 890.01 
Table 1: Physical data of the title compounds

\begin{tabular}{|c|c|c|c|c|c|c|c|}
\hline S. No. & Compounds number & $\mathbf{R}$ & Color & M.F & M.W & M.P $\left({ }^{\circ} \mathrm{C}\right)$ & UV \\
\hline 1 & $2 \mathrm{a}$ & $4-\mathrm{F}$ & Cream & $\mathrm{C}_{17} \mathrm{H}_{12} \mathrm{FNO}_{3}$ & 297.28 & $126-128$ & 225 and 229.2 \\
\hline 2 & $2 b$ & $4-\mathrm{NO}_{2}$ & Orange & $\mathrm{C}_{17}^{17} \mathrm{H}_{12}^{12} \mathrm{~N}_{3} \mathrm{O}_{5}^{3}$ & 338.29 & $136-138$ & 302 and 302.8 \\
\hline 3 & $2 \mathrm{c}$ & $4-\mathrm{OH}^{2}$ & Orange & $\mathrm{C}_{17} \mathrm{H}_{13} \mathrm{NO}_{4}^{3}$ & 295.29 & $122-124$ & 345 \\
\hline 4 & $2 d$ & $3,4,5-\left(\mathrm{OCH}_{3}\right)_{3}$ & Cream & $\mathrm{C}_{20}^{17} \mathrm{H}_{19}^{13} \mathrm{NO}_{6}^{4}$ & 369.37 & $140-142$ & 238 and 263.5 \\
\hline 5 & $2 \mathrm{e}$ & $4-\mathrm{N}\left(\mathrm{CH}_{3}\right)_{2}$ & Cream & $\mathrm{C}_{19}^{20} \mathrm{H}_{18} \mathrm{~N}_{2} \mathrm{O}_{3}$ & 322.36 & $151-153$ & 296 and 229.7 \\
\hline 6 & $2 f$ & $4-\mathrm{N}\left(\mathrm{C}_{2} \mathrm{H}_{5}\right)_{2}^{2}$ & Green & $\mathrm{C}_{21} \mathrm{H}_{22} \mathrm{~N}_{2} \mathrm{O}_{3}$ & 350.41 & $155-157$ & 294 and 294.8 \\
\hline
\end{tabular}

Table 2: Antibacterial activity (zone of inhibition)

\begin{tabular}{|c|c|c|c|c|}
\hline S. No. & Compound & $\mathbf{R}$ & Concentration $(\mu \mathrm{g} / \mathrm{ml})$ & Zone of inhibition $(\mathrm{mm})$ \\
\hline \multirow[t]{2}{*}{2} & $2 \mathrm{a}$ & $4-\mathrm{F}$ & 50 & $12 \pm 0.2$ \\
\hline & & & 100 & $14 \pm 0.4$ \\
\hline \multirow[t]{2}{*}{4} & $2 b$ & $4-\mathrm{NO}_{2}$ & 50 & $8 \pm 0.2$ \\
\hline & & & 100 & $9 \pm 0.5$ \\
\hline \multirow[t]{2}{*}{5} & $2 c$ & $4-\mathrm{OH}$ & 50 & $8 \pm 0.2$ \\
\hline & & & 100 & $8 \pm 0.4$ \\
\hline \multirow[t]{2}{*}{6} & $2 \mathrm{~d}$ & $3,4,5-\left(\mathrm{OCH}_{3}\right)_{2}$ & 50 & $16 \pm 0.6$ \\
\hline & & & 100 & $19 \pm 0.8$ \\
\hline \multirow[t]{2}{*}{7} & $2 \mathrm{e}$ & 4-N $\left(\mathrm{CH}_{3}\right)_{2}$ & 50 & $5 \pm 0.2$ \\
\hline & & & 100 & $6 \pm 0.2$ \\
\hline \multirow[t]{2}{*}{8} & $2 \mathrm{f}$ & $4-\mathrm{N}\left(\mathrm{C}_{2} \mathrm{H}_{5}\right)_{2}$ & 50 & $5 \pm 0.3$ \\
\hline & & & 100 & $7 \pm 0.5$ \\
\hline Amikacin & -- & -- & & $28 \pm 1.2$ \\
\hline
\end{tabular}

Table 3: Molecular properties of 4-substituted benzylidene-2-(phenoxymethyl) oxazol-5 (4H)-ones

\begin{tabular}{|c|c|c|c|c|c|c|c|c|c|}
\hline Compound number & $\mathbf{R}$ & $\operatorname{miLog} P$ & TPSA & MW & nON & $\begin{array}{l}\text { nOH } \\
\text { NH }\end{array}$ & Nviol & Nrotb & MV \\
\hline $2 a$ & $4-\mathrm{F}$ & 3.45 & 52.34 & 297.29 & 4 & 0 & 0 & 4 & 253.97 \\
\hline $2 b$ & $4-\mathrm{NO}_{2}$ & 3.24 & 98.16 & 324.29 & 7 & 0 & 0 & 5 & 272.38 \\
\hline $2 c$ & $4-\mathrm{OH}^{2}$ & 2.69 & 72.56 & 259.29 & 5 & 1 & 0 & 4 & 257.06 \\
\hline $2 d$ & $3,4,5-\left(\mathrm{OCH}_{3}\right)_{3}$ & 2.92 & 80.04 & 369.37 & 7 & 0 & 0 & 7 & 325.68 \\
\hline $2 \mathrm{e}$ & $4-\mathrm{N}\left(\mathrm{CH}_{3}\right)_{2}$ & 3.39 & 55.58 & 322.36 & 5 & 0 & 0 & 5 & 294.95 \\
\hline $2 \mathrm{f}$ & 4-N $\left(\mathrm{C}_{2} \mathrm{H}_{5}\right)_{2}$ & 4.14 & 55.58 & 350.42 & 5 & 0 & 0 & 7 & 328.55 \\
\hline
\end{tabular}

miLogP - (Lipophilicity); TPSA: Topological surface area, MW: Molecular weight, ON - Sum of hydrogen bond receptors, OHNH: Sum of hydrogen bond donors, Nviol: Number of Violations, Nrotb: Number of rotatable bonds, MV: Molecular volume

Table 4: Bioactivity scores of 4-substituted benzylidene-2-(phenoxymethyl) oxazol-5 (4H)-ones

\begin{tabular}{|c|c|c|c|c|c|c|c|}
\hline $\begin{array}{l}\text { Compound } \\
\text { number }\end{array}$ & $\mathbf{R}$ & $\begin{array}{l}\text { GPCR } \\
\text { ligand }\end{array}$ & $\begin{array}{l}\text { Ion channel } \\
\text { modulator }\end{array}$ & $\begin{array}{l}\text { Kinase } \\
\text { inhibitor }\end{array}$ & $\begin{array}{l}\text { Nuclear } \\
\text { receptor ligand }\end{array}$ & $\begin{array}{l}\text { Protease } \\
\text { inhibitor }\end{array}$ & $\begin{array}{l}\text { Enzyme } \\
\text { inhibitor }\end{array}$ \\
\hline $2 a$ & $4-F$ & -0.69 & -1.02 & -0.51 & -0.51 & -0.75 & -0.43 \\
\hline $2 b$ & $4-\mathrm{NO}_{2}$ & -0.78 & -0.99 & -0.63 & -0.58 & -0.78 & -0.50 \\
\hline $2 c$ & $4-\mathrm{OH}^{2}$ & -0.40 & -0.61 & -0.10 & -0.40 & -0.42 & -0.12 \\
\hline $2 \mathrm{~d}$ & $3,4,5-(\mathrm{OCH} 3)_{3}$ & -0.61 & -0.90 & -0.45 & -0.52 & -0.64 & -0.38 \\
\hline $2 \mathrm{e}$ & 4-N $\left(\mathrm{CH}_{3}\right)_{2}$ & -0.62 & -0.96 & -0.45 & -0.46 & -0.66 & -0.40 \\
\hline $2 f$ & 4-N $\left(\mathrm{C}_{2} \mathrm{H}_{5}\right)_{2}$ & -0.55 & -0.90 & -0.45 & -0.44 & -0.62 & -0.42 \\
\hline
\end{tabular}

GPCR: G-protein coupled receptor

(C-H aromatic bend). ${ }^{1} \mathrm{H}$ NMR $\left(\mathrm{CDCl}_{3}\right): \delta 54.12\left(\mathrm{~s}, 2 \mathrm{H},-\mathrm{OCH}_{2}-\right), 0.01$ (s, 3H, OH) 6.42-7.56 (m, 10H, Ar-H\&=CH-) MS (m/z): $295.6[\mathrm{M}]^{+}$.

2d: 2-(phenoxymethyl)-4-(3, 4, 5-trimethoxybenzylidene) oxazol5(4H)-one

FTIR (KBr) cm ${ }^{-1}: 2958.47$ (C-H aromatic str), $2904.24 \quad(\mathrm{C}-\mathrm{H}$ aliphatic str), 1750.87 ( $\mathrm{C}=0$ str of lactone ring), 1668.57 ( $\mathrm{C}=\mathrm{C}$ str), $890.68\left(\mathrm{C}-\mathrm{H}\right.$ aromatic bend). ${ }^{1} \mathrm{H}$ NMR $\left(\mathrm{CDCl}_{3}\right): \delta 3.09\left(\mathrm{~s}, 3 \mathrm{H}, \mathrm{OCH}_{3}\right)$ $3.11\left(\mathrm{~s}, 6 \mathrm{H},\left(\mathrm{OCH}_{3}\right)_{2} 4.12\left(\mathrm{~s}, 2 \mathrm{H},-\mathrm{OCH}_{2}-\right), 6.91-7.88(\mathrm{~m}, 8 \mathrm{H}, \mathrm{Ar}-\mathrm{H} \&=\mathrm{CH}-)\right.$ MASS (m/z): $370.14[\mathrm{M}+1]^{+}$.

2e: 4-(4-(dimethylamino) benzylidene)-2-(phenoxymethyl) oxazol5(4H)-one

FTIR (KBr) cm ${ }^{-1}: 3058.24$ (C-H aromatic str), 2904.50 (C-H aliphatic str), 1741.28 ( $\mathrm{C}=0$ str of lactone ring), 1656.32 ( $\mathrm{C}=\mathrm{C}$ stretch), 865.29
(C-H aromatic bend).). ${ }^{1} \mathrm{H}$ NMR $\left(\mathrm{CDCl}_{3}\right): \delta 3.89\left(\mathrm{~s}, 6 \mathrm{H}, \mathrm{N}\left(\mathrm{CH}_{3}\right)_{2} 4.09\right.$ (s, 2H, $-\mathrm{OCH}_{2}-$ ) $6.93-7.89(\mathrm{~m}, 10 \mathrm{H}, \mathrm{Ar}-\mathrm{H} \&=\mathrm{CH}-)$ MS (m/z): $322.4[\mathrm{M}]^{+}$.

2f: 4-(4-(diethylamino) benzylidene)-2-(phenoxymethyl) oxazol5(4H)-one

FTIR (KBr) cm ${ }^{-1}: 2989.68$ (C-H aromatic str), 2897.08 (C-H aliphatic str), 1724.53 ( $\mathrm{C}=0$ stretch of lactone ring), 1663.85 ( $\mathrm{C}=\mathrm{C}$ str), 874.15 (C-H aromatic bend). ${ }^{1} \mathrm{H}$ NMR $\left(\mathrm{CDCl}_{3}\right): \delta 1.68-1.73\left[\mathrm{t}, 6 \mathrm{H},\left(\mathrm{CH}_{3}\right)_{2}\right] 3.42$ (q, 4H, $\mathrm{NCH}_{2}\left(\mathrm{CH}_{3}\right)_{2}, 4.12\left(\mathrm{~s}, 2 \mathrm{H},-\mathrm{OCH}_{2}-\right), 7.01-7.88(\mathrm{~m}, 10 \mathrm{H}, \mathrm{Ar}-\mathrm{H} \&=\mathrm{CH}-)$ MS (m/z): 350.5[M] .

Antibacterial activity

The title compounds (2a-2f) were tested for their antibacterial activity against E. coli. Compounds were dissolved in DMSO and used at a concentration of $50 \mu \mathrm{g}$ and $100 \mu \mathrm{g} / \mathrm{ml}$. The activity was recorded as a zone of inhibition after the incubation period of $24 \mathrm{~h}$ at $37^{\circ} \mathrm{C}$. 
The data showed 3, 4, 5- trimethoxy derivative ( $2 \mathrm{~d}$ ) as potent derivative among all the compounds which showed the zone of inhibition of $19 \mathrm{~mm}$ at $100 \mu \mathrm{g} / \mathrm{ml}$ and $16 \mathrm{~mm}$ at $50 \mu \mathrm{g} / \mathrm{ml}$, respectively (Fig. 6). In the case of 4-substituted benzylidene-2-(phenyl) oxazol-5(4H)-ones, it was observed that methoxy substitution on benzylidene is favorable for antibacterial activity against Gram-negative as well as Gram-positive organisms [18]. Similarly, in the present study, methoxy functionality is found to be favorable. However, the potency of $2 \mathrm{~d}$ is less than that of amikacin, a standard drug which exhibited $28 \mathrm{~mm}$ of the zone of inhibition. The fluoro derivative (2a) also showed significant inhibition with the zone of inhibition of $14 \mathrm{~mm}$ at $100 \mu \mathrm{g} / \mathrm{ml}$ concentration and $12 \mathrm{~mm}$ at $50 \mu \mathrm{g} / \mathrm{ml}$. Introduction of fluorine enhances the lipophilicity of the molecule and also creates a dipole charge between carbon and fluorine. Lipophilicity and dipole charges increase the ability of a compound to form various interactions at the target binding site. 4-Hydroxy, 4-dimethylamino and 4-diethyl amino derivatives (2c, 2e, and $2 \mathrm{f}$ ) were unable to exert any significant activity against $E$. coli (Table 2). The poor activity of 2c, 2e, and $2 \mathrm{f}$ suggested that electron releasing substituent groups are not significantly contributing to the activity.

These observations indicated that 4-fluoro and 3, 4, 5-trimethoxy substitution on benzylidene ring significantly favor antibacterial activity against $E$. coli. In summary, it can be suggested that the introduction of phenoxy methylene linker in the oxazoles-5-one structure is a good strategy to develop antibacterial agents.

\section{Prediction of molecular properties}

All the compounds were predicted to have good oral bioavailability as their molecular weight is $<500$ (297-369), hydrogen bond donors are $<5(0)$, HBA are $<10(4-7)$, and no of rotatable bonds are $<10(4-7)$ (Tables 3 and 4). Pharmacokinetic properties prediction showed that their absorption in the gastrointestinal tract will be good (Table 5).

Lipinski rule: miLog $\mathrm{p} \leq 5, \mathrm{MW} \leq 500, \mathrm{HBA} \leq 10$ and $\mathrm{HBD} \leq 5$.

\section{Molecular docking studies}

Fluoroquinolones and coumarin derivatives are potent antimicrobial agents which act by DNA Gyrase inhibition. DNA Gyrase with its subunits (2 Gyr A and 2 Gyr B) introduces negative supercoils in DNA and effectively inhibits initiation step of DNA synthesis [31]. Interestingly, several potent inhibitors such as novobiocin, clorobiocin, cyclothialidine, and 5 '-adenylyl- $\beta, \gamma$-imidodiphosphate (ATP analog) were found to interact with Asp-73, crucial amino acid present in the ATP binding domain of DNA Gyrase [32]. Basarab et al. synthesized spiropyrimidinetriones with a benzisoxazole heterocyclic scaffold and reported that these derivatives possess potent DNA Gyrase inhibitory activity [33]. Literature findings revealed that various oxazolones derivatives exhibit potent antimicrobial activity against Gramnegative and Gram-positive organisms. Previously, molecular docking studies were performed for a series of 4-benzylidene-2-(4-hydroxy-3methoxystyryl) oxazol-5(4H)-ones and the binding poses revealed that these molecules were able to interact with Asp-73 and Arg-176 amino acids [34]

Molecular docking studies were performed to explore binding affinities of synthesized compounds with the target enzyme.

4-Substituted benzylidene-2-(phenoxymethyl) oxazol-5(4H)-ones In the series of 4-substituted benzylidene-2-(phenoxymethyl) oxazol$5(4 \mathrm{H})$-ones, 3, 4, 5-trimethoxy derivative, $2 \mathrm{~d}$, exhibited good interaction
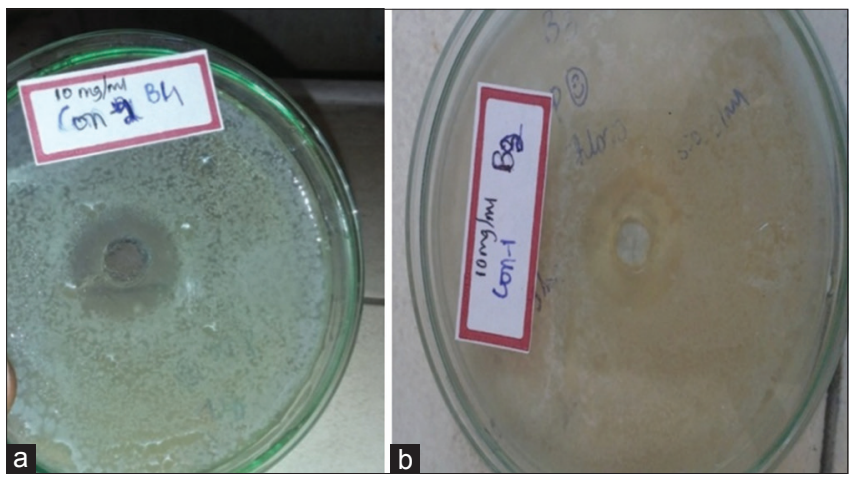

Fig. 6: ( $a$ and $b$ ) Zone of inhibition of $2 a$ and $2 d$

Table 5: ADME parameters of 4-substituted benzylidene-2-(phenoxymethyl) oxazol-5 (4H)-ones

\begin{tabular}{|c|c|c|c|c|c|c|c|}
\hline \multirow{2}{*}{$\begin{array}{l}\text { Compound } \\
\text { number }\end{array}$} & \multirow[t]{2}{*}{$\mathbf{R}$} & \multicolumn{2}{|c|}{ Absorption } & \multirow[t]{2}{*}{ PPB } & \multirow[t]{2}{*}{ BBB } & \multirow[t]{2}{*}{ SKIN } & \multirow[t]{2}{*}{ MDCK } \\
\hline & & HIA & $\mathrm{CaCo} 2$ & & & & \\
\hline $2 a$ & $4-\mathrm{F}$ & 97.83 & 35.37 & 92.73 & 1.77 & -3.11 & 21.32 \\
\hline $2 b$ & $4-\mathrm{NO}_{2}$ & 95.50 & 14.75 & 92.36 & 0.01 & -3.00 & 237.62 \\
\hline $2 c$ & $4-\mathrm{OH}^{2}$ & 96.41 & 21.80 & 92.76 & 0.25 & -3.00 & 29.26 \\
\hline $2 \mathrm{~d}$ & $3,4,5-(\mathrm{OCH} 3)_{3}$ & 96.63 & 51.93 & 84.67 & 0.03 & -3.39 & 142.40 \\
\hline $2 \mathrm{e}$ & $4-\mathrm{N}\left(\mathrm{CH}_{3}\right)_{2}$ & 95.87 & 54.71 & 93.34 & 0.69 & -3.06 & 158.75 \\
\hline $2 \mathrm{f}$ & 4-N $\left(\mathrm{C}_{2} \mathrm{H}_{5}\right)_{2}$ & 96.06 & 56.19 & 93.57 & 0.55 & -2.79 & 105.67 \\
\hline
\end{tabular}

CaCo2: CaCo2 cell permeability, HIA: Human intestinal absorption, PPB: Plasma protein binding, BBB: Blood-brain barrier penetration, SKIN: Skin permeability, MDCK:

Maden Darby canine kidney cell permeability. ADME: Absorption, distribution, metabolism, and excretion

Table 6: Docking results for 4-Substituted benzylidene-2-(phenoxymethyl) oxazol-5 (4H)-ones

\begin{tabular}{|c|c|c|c|c|c|}
\hline S. No. & $\mathbf{R}$ & $\operatorname{Energy}(\Delta G)$ & Full fitness & Interacting amino acids & Bond distance $\left(\mathrm{A}^{0}\right)$ \\
\hline \multirow[t]{2}{*}{$2 \mathrm{a}$} & $4-\mathrm{F}$ & -7.82 & -1291.94 & Thr-165 H-Lig 0 & 2.41 \\
\hline & & & & Gly-77 NH- Lig 0 & 2.34 \\
\hline $2 b$ & $4-\mathrm{NO}_{2}$ & -8.05 & -1270.80 & Asn-46 H-Lig 0 & 2.62 \\
\hline \multirow[t]{2}{*}{$2 c$} & $4-\mathrm{OH}^{2}$ & -7.95 & -1306.97 & Asn- 46 H-Lig 0 & 2.62 \\
\hline & & & & Arg-136 H-Lig 0 & 2.16 \\
\hline $2 \mathrm{~d}$ & $3,4,5-\mathrm{OCH}_{2}$ & -8.41 & -1265.74 & Arg-76 H-Lig O & 2.51 \\
\hline $2 \mathrm{e}$ & $4-\mathrm{N}\left(\mathrm{CH}_{3}\right)_{2}^{3}$ & -7.94 & -1293.08 & Asn-46 H-Lig 0 & 2.87 \\
\hline $2 \mathrm{f}$ & 4-N $\left(\mathrm{C}_{2} \mathrm{H}_{5}\right)_{2}$ & -7.80 & -1286.35 & Asn-46 H-Lig O & 2.25 \\
\hline \multirow[t]{3}{*}{ Std } & - & -7.91 & -1073.85 & Asp-73 O-Lig H & 1.88 \\
\hline & & & & Gly-77 NH-Lig O & 2.30 \\
\hline & & & & Thr-165 H-LigO & 2.16 \\
\hline
\end{tabular}

Std: Ciprofloxacin 
Table 7: Docking results for 4-substituted benzylidene-2-(phenyl) oxazol-5 (4H)-ones

\begin{tabular}{llllll}
\hline S. No. & $\mathbf{R}$ & Energy $(\boldsymbol{\Delta G})$ & Full fitness & Interacting amino acids & Bond distance $\left(\mathbf{A}^{\mathbf{}}\right)$ \\
\hline 1 & $4-\mathrm{F}$ & -7.40 & -1290.92 & Gly-77 NH-Lig N & 2.17 \\
& & & Thr-165 H-Lig O & 2.06 \\
2 & $4-\mathrm{NO}_{2}$ & -7.64 & -1279.82 & Arg-136 H-Lig O & 1.92 \\
& & & Asn-46 H-Lig O & 2.58 \\
3 & $4-\mathrm{OH}$ & -7.68 & -1301.43 & Asp-73 O-Lig H & 2.88 \\
4 & $3,4,5-\mathrm{OCH}_{3}$ & -8.11 & -1264.53 & Asn-46 H-Lig O & 2.65 \\
5 & $4-\mathrm{N}\left(\mathrm{CH}_{3}\right)_{2}$ & -7.86 & -1212.35 & Asn-46 H-Lig O & 2.66 \\
6 & $4-{\mathrm{N}\left(\mathrm{C}_{2} \mathrm{H}_{5}\right)_{2}}$ & -7.60 & -1292.19 & Asn-46 H-Lig O & \\
\hline
\end{tabular}

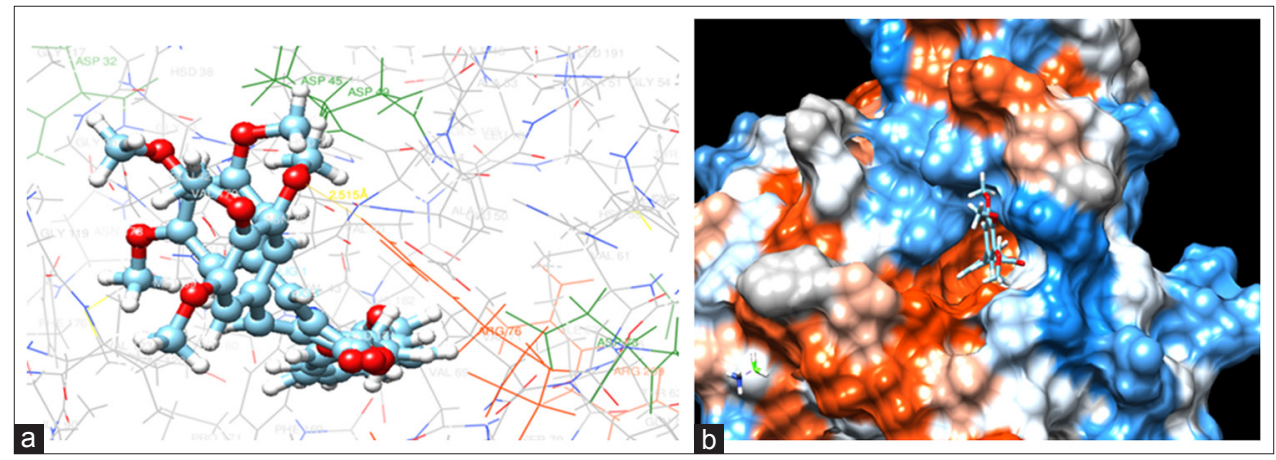

Fig. 7 (a) Compound 2d interaction with amino acids, (b) hydrophobic mode

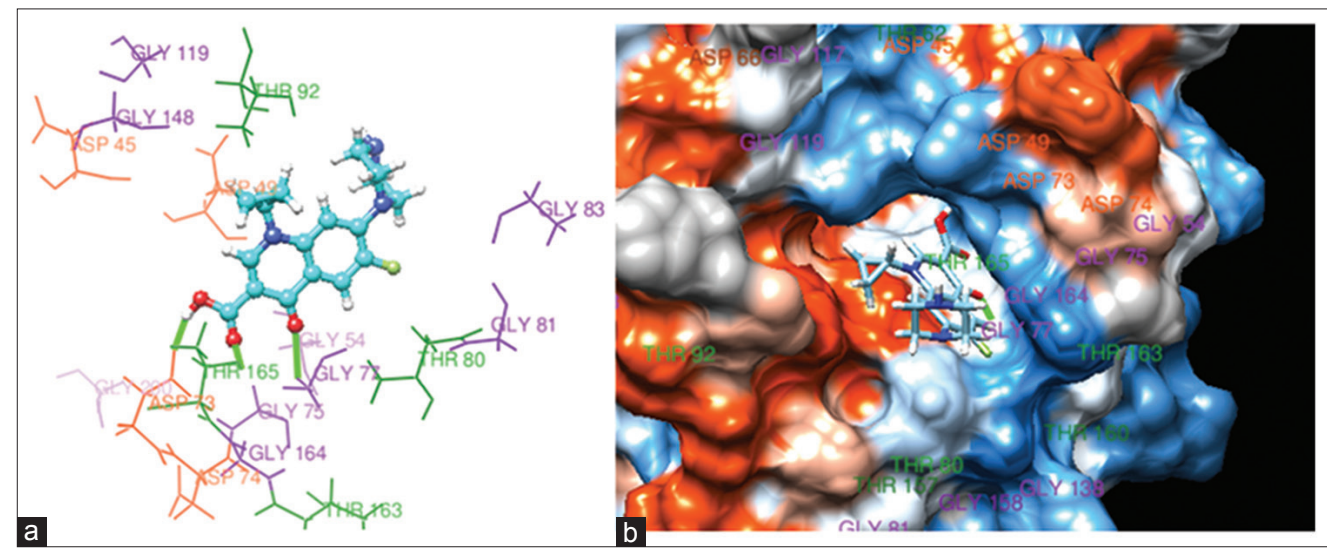

Fig. 8: (a) Binding mode of ciprofloxacin in the active site of DNA gyrase, (b) hydrophobic mode

with DNA-Gyrase enzyme ( $\Delta \mathrm{G}$ of $-8.41 \mathrm{kcal} / \mathrm{mol})$. Abolhasani et al. mentioned the importance of methoxy functionality and substitution pattern of methoxy groups at the target binding site [35]. Moderate binding interactions were observed for all the other derivatives indicating that their presence enhances the affinity of the molecule for the target binding site. Ciprofloxacin showed good interaction with $-7.91 \mathrm{kcal} / \mathrm{mol}$ of Gibbs free energy (Table 6).

To compare the influence of oxymethylene linker, SwissDock studies were performed using a set of 4-substituted benzylidene-2-(phenyl) oxazol-5(4H)-ones (1-6) with the same substitution as of 4-substituted benzylidene-2-(phenyl) oxazol-5(4H)-ones (2a-2f).

\section{4-Substituted benzylidene-2-(phenyl) oxazol-5(4H)-ones}

Results showed that Gibbs free energy $(\Delta G)$ values for 4-substituted benzylidene-2-(phenyl) oxazol-5(4H)-ones were found to be in the range of $-7.40 \mathrm{kcal} / \mathrm{mol}$ to $-8.11 \mathrm{kcal} / \mathrm{mol}$ whereas for 4-substituted benzylidene-2-(phenoxymethyl) oxazol-5(4H)ones, the values were found to be from $-7.82 \mathrm{kcal} / \mathrm{mol}$ to -8.41 $\mathrm{kcal} / \mathrm{mol}$. Replacement of phenyl ring with conformationally flexible phenoxy methylene moiety augments binding interactions with the target enzyme. Results showed that 3, 4, 5-trimethoxy derivative (4) demonstrated binding affinity of $-8.11 \mathrm{kcal} / \mathrm{mol}$ and other derivatives also showed good interactions with the active site (Table 7).

There is no similarity in the binding poses of ciprofloxacin and the synthesized compounds except in the case of 2a; ciprofloxacin formed three hydrogen bonds with Asp-73, Gly-77, and Thr-165 (Fig. 7) and 2a similarly found to interact with Gly-77 and Thr-165. All the other compounds $2 \mathrm{~b}-2 \mathrm{f}$ were situated near Asn- 46 and formed hydrogen bonding with this amino acid. Compound $2 \mathrm{~d}$ formed hydrogen bonding interactions with Arg-76 (Figs. 7 and 8). Binding orientations of 4-substituted benzylidene-2-(phenyl) oxazol-5(4H)-ones were almost identical to that of 4-substituted benzylidene-2-(phenoxymethyl) oxazol-5(4H)-ones where 4-fluoro substituted derivative showed hydrogen bonding with Gly-77 and Thr-165 and other derivatives were bonded with Asn-46.

The correlation was observed between molecular docking studies and in vitro antibacterial activity in case of active compound $2 \mathrm{~d}$, which showed high activity against $E$. coli (zone of inhibition $19 \mathrm{~mm}$ at $100 \mu \mathrm{g} / \mathrm{ml})$ and good interactions with DNA-Gyrase enzyme $(\Delta \mathrm{G}$ of $-8.41 \mathrm{kcal} / \mathrm{mol})$. 


\section{CONCLUSION}

A series of 4-substituted benzylidene-2-phenyloxazol-5(4H)-ones were synthesized in good yields by applying the mechanochemical stirring approach. It was interesting to observe that the insertion of oxymethylene linker between 1,3-oxazol-5-one scaffold and phenyl ring; a simple structural modification increased the potentiality of compounds. 4-Fluoro substitution (2a) and 3, 4, 5-trimethoxy substitution (2d) on benzylidene ring significantly enhanced the antibacterial activity against $E$. coli. High activity of active compounds might be due to their good binding affinity toward the target enzyme DNA gyrase. The data regarding molecular and ADME properties indicate druggability of the title compounds.

\section{ACKNOWLEDGMENTS}

The authors are very thankful to DST-CURIE (Consolidation of University Research for Innovation and Excellence in Women Universities) SPMVV, Tirupati, for providing FTIR spectral data of the synthesized compounds.

\section{AUTHORS' CONTRIBUTIONS}

All authors have made considerable contributions to the work reported in the manuscript. Maneesha M, Ome Shanthi T, and Satya varalakshmi D synthesized and screened the compounds for antibacterial activity. Manjusha RK performed molecular docking study using SwissDock and other in silico studies mentioned in the manuscript. Shaheen Begum and Arifa Begum prepared the manuscript and Bharathi K supervised and reviewed the work.

\section{CONFLICTS OF INTEREST}

The authors confirm that this article content has no conflicts of interest.

\section{REFERENCES}

1. Mukherjee S. Emerging infectious diseases: Epidemiological perspective. Indian J Dermatol 2017;62:459-67.

2. Noel GJ, Draper MP, Hait H, Tanaka SK, Arbeit RD. A randomized, evaluator-blind, phase 2 study comparing the safety and efficacy of omadacycline to those of linezolid for treatment of complicated skin and skin structure infections. Antimicrob Agents Chemother 2012;56:5650-4.

3. Lin J, Nishino K, Roberts MC, Tolmasky M, Aminov RI, Zhang L, et al. Mechanisms of antibiotic resistance. Front Microbiol 2015;6:1-3.

4. Brown ED, Wright GD. Antibacterial drug discovery in the resistance era. Nature 2016;529:336-43.

5. Chandra H, Bishnoi P, Yadav A, Patni B, Mishra AP, Nautiyal AR, et al. Antimicrobial resistance and the alternative resources with special emphasis on plant-based antimicrobials-a review. Plants 2017;6:1-11.

6. Haque M. Antimicrobial use, prescribing, and resistance in selected ten selected developing countries: A brief overview. Asian J Pharm Clin Res 2017;10:37-45.

7. El-Gamal KM, Sherbiny FF, El-Morsi AM, Abu-El-Khair HE, Eissa IH, El-Sebaei MM. Design, synthesis and antimicrobial evaluation of some novel quinoline derivatives. Pharm Pharmacol Int J 2015;2:165-77.

8. Onkol T, Doğruer DS, Uzun L, Adak S, Ozkan S, Sahin MF, et al. Synthesis and antimicrobial activity of new 1,2,4-triazole and 1,3,4-thiadiazole derivatives. J Enzyme Inhib Med Chem 2008;23: $277-84$.

9. El-Salam NM, Mostafa MS, Ahmed GA, Alothman OY. Synthesis and antimicrobial activities of some new heterocyclic compounds based on 6-chloropyridazine-3 (2H) -thione. J Chem 2013;13:1-8.

10. Sharma R. Review: Antimicrobial agents based on nitrogen and sulfur containing heterocycles. Asian J Pharm Clin Res 2017;10:47-9.

11. Rajitha G, Prasad KV, Bharathi K. Synthesis and biological evaluation of 3-amino pyrazolones. Asian J Chem 2011;23:684-6.

12. Bala S, Saini M, Sunil K. Methods for synthesis of oxazolones: A review. Int J Chemtech Res 2011;3:1102-18.
13. Sharada LN, Aparna Y, Saba M, Sunitha SN, Viveka L. A review on reactions and applications of oxazolones. Int J Sci Res 2015;5:1-9.

14. El-Mekabaty A. Erlenmeyer azlactones: Synthesis, reactions and biological activity. Int J Mod Org Chem 2013;2:40-66.

15. Mariappan G, Saha BP, Datta S, Kumar D, Haldar PK. Design, synthesis and antidiabetic evaluation of oxazolone derivatives. J Chem Sci 2011;123:335-41.

16. Al-Ghabkari A, Deng JT, McDonald PC, Dedhar S, Alshehri M, Walsh MP, et al. A novel inhibitory effect of oxazol-5-one compounds on ROCKII signaling in human coronary artery vascular smooth muscle cells. Sci Rep 2016;6:32118.

17. Jat LR, Mishra R, Pathak D. Synthesis and anticancer activity of 4-benzylidene-2- phenyloxazol-5(4h)-one derivatives. Int $\mathrm{J}$ Pharm Pharm Sci 2012;4:378-80.

18. Saravanan VS, Selvan PS, Gopal N, De B, Gupta JK. Synthesis and antibacterial activity of some 4-substituted-2-phenyl oxazol-5(4H)-one derivatives. Asian J Chem 2006;18:2511-4.

19. Bayoumi WA, Shaymaa HA, Mohamed ES. Synthesis and evaluation of new 2-iminothiazolidin-4-one and thiazolidin-2, 4-dione derivatives as antimicrobial and anti-inflammatory agents. Open Chem J 2014;1:33-8.

20. Ottana R, Maccari R, Barreca ML, Bruno G, Rotondo A, Rossi A, et al. 5-Arylidene-2-imino-4-thiazolidinones: Design and synthesis of novel anti-inflammatory agents. Bioorg Med Chem 2005;13:134243-52.

21. Vicini P, Zani F, Cozzini P, Doytchinova I. Hydrazones of 1,2-benzisothiazole hydrazides: Synthesis, antimicrobial activity and QSAR investigations. Eur J Med Chem 2002;37:553-64.

22. Amjid I, Hamid LS, Ashraf CM, Ahmad M, Weaver GW. Synthesis, characterization and antibacterial activity of azomethine derivatives derived from 2-formylphenoxyacetic acid. Molecules 2007;12:245-54.

23. Pattan SR, Hullolikar RL, Pattan JS, Kapadnis BP, Dighe NS, Dengale SS. Synthesis and evaluation of some new pyrazolo phenoxy acetic acid derivatives for their antitubercular activity. J Pharm Sci Res 2009;1:63-8

24. Aaglawe MJ, Dhule SS, Bahekar SS, Wakte PS, Shinde DB. Synthesis and antibacterial activity of some oxazolone derivatives. J Korean Chem Soc 2003;47:133-6.

25. Varma RS. Greener chemical syntheses using mechanochemical mixing or microwave and ultrasound irradiation. Green Chem Lett Rev 2007; $1: 37-45$

26. Leonardi M, Villacampa M, Menéndez JC. Multicomponent mechanochemical synthesis. Chem Sci 2018;9:2042-64.

27. El-Sayed AA, Hemdan MM, Fahmy AF. Multicomponent synthesis of 4-arylidene-2-phenyl-5(4H)-oxazolones (azlactones) using a mechanochemical approach. Chem Cent J 2016;10:1-7.

28. Rajitha G, Prasad KV, Bharathi K. Synthesis and evaluation of substituted cinnamoyl alanines for antiinflammatory, analgesic and antioxidant activities. Asian J Chem 2010;22:1197-04.

29. Grosdidier A, Zoete V, Michielin O. SwissDock, a protein-small molecule docking web service based on EADock DSS. Nucleic Acids Res 2011;39:W270-7.

30. Soujanya M, Rajitha G. Microwave assisted synthesis, characterization, molecular docking and antimicrobial evaluation of 4-nitrocinnamide analogues. Int J Pharm Sci Res 2017;8:3786-94.

31. Walker SS, Labroli M, Painter RE, Wiltsie J, Sherborne B, Murgolo N, et al. Antibacterial small molecules targeting the conserved TOPRIM domain of DNA gyrase. PLoS One 2017;12:e0180965.

32. Mayer C, Janin YL. Non-quinolone inhibitors of bacterial Type IIA topoisomerases: A feat of bioisosterism. Chem Rev 2014;114:2313-42.

33. Basarab GS, Brassil P, Doig P, Galullo V, Haimes HB, Kern G. Novel DNA gyrase inhibiting spiropyrimidinetriones with a benzisoxazole scaffold: SAR and in vivo characterization. J Med Chem 2014;57: 9078-95.

34. Manjusha RK, Begum A, Swapna B, Begum S, Bharathi K, Sujatha D. Molecular docking studies of 4-benzylidene-2-(4hydroxy-3- methoxystyryl) oxazol-5(4H)-ones; Interactions with Arg136 and Asp-73 of DNA gyrase. J Glob Trends Pharm Sci 2018;9: $5172-80$.

35. Abolhasani H, Zarghi A, Hamzeh-Mivehroud M, Alizadeh AA, Mojarrad SD. In-silico investigation of tubulin binding modes of a series of novel antiproliferative spiroisoxazoline compounds using docking studies. Iran J Pharm Res 2015;14:141-7. 